'Departamento de Medicina

Interna, Hospital Italiano de

Buenos Aires, Argentina.

2Sección Hematología,

departamento de Medicina

Interna, Hospital Italiano de

Buenos Aires, Argentina.

${ }^{3}$ Área de Investigación en

Medicina Interna, departamento de Medicina Interna, Hospital

Italiano de Buenos Aires, Argentina.

Conflictos de interés: Los autores refieren no presentar conflictos de interés que declarar.

Recibido el 24 de agosto de 2016, aceptado el 6 de marzo de 2017.

Corresponenicia a: Dr. Warley Fernando Hospital Italiano de Buenos Aires, Tte. Gral. J. D. Perón 4190, Buenos Aires, Argentina. ferwarley@gmail.com

\section{Características clínicas y mortalidad de pacientes adultos con síndrome hemafagocítico, estudio de cohorte retrospectiva}

\author{
FERNANDO WARLEY ${ }^{1,3}$, BELÉN M. BONELLA ${ }^{1,3}$, \\ M. SILVINA ODSTRCIL-BOBILLO ${ }^{1,3}$, VICTORIA OTERO ${ }^{2}$, \\ GABRIEL WAISMAN ${ }^{1}$, GISELA BENDELMAN ${ }^{1,3}$, DIEGO GIUNTA ${ }^{3}$, \\ VERÓNICA PEUCHOT ${ }^{3}$, CATALINA M. UNGARO ${ }^{1,3}$
}

\section{Hemophagocytic lymphohistiocytosis. Experience in 27 patients}

Background: Hemophagocytic lymphohistiocytosis (HLH) is an aggressive and life-threatening syndrome of excessive immune activation Aim: To describe the clinical characteristics, causes and survival associated with HLH. Material and Methods: Review of medical records of patients with HLH attended between 2004 and 2016. They were classified according to their probable cause in: associated with immunosuppression, cancer, post-infectious or idiopathic. Kaplan-Meier survival analysis was performed. Results: Twenty seven patients with HLH aged 18 to 87 years (59\% men), were detected. Fourteen (52\%) were secondary to immunosuppression, six (22\%) were post-infectious, five (18\%) were associated with cancer and two (7\%) were of unknown cause. There were no significant differences in clinical or laboratory features between these etiologies. Within the immunosuppressed group, 12 (86\%) were patients with oncologic or hematologic diseases or bone marrow transplantation. Associated cancers were mostly oncohematologic diseases. Thirty-day mortality was $53.4 \%$ (95\% confidence intervals (CI) 32.7-70.3\%), despite the treatment. Mortality was significantly associated with the presence of renal failure with a hazard ratio (HR) of 3.4 (95\% CI of 1.2-9.9, $p=0.025)$. Treatment of the underlying disease proved to be protective against mortality with an HR of $0.3(95 \%$ CI 0.1 to $0.98, p=0.046)$. Conclusions: The prognosis of HLH could be related to the treatment of the underlying disease. The study of the pathophysiology of this syndrome will allow a better understanding and treatment.

(Rev Med Chile 2017; 145: 344-350)

Key words: Hemaphagocytic; Immunosuppression; Immunocompromised Host; Lymphohistiocytosis.
L a linfohistiocitosis hemofagocítica o síndrome hemofagocítico (SHF) se caracteriza por la activación del sistema inmunológico de manera exacerbada e inefectiva. Una de sus principales componentes es la infiltración de linfocitos y macrófagos con actividad hemofagocítica en diferentes órganos, especialmente el hígado, bazo, médula ósea y sistema nervioso central ${ }^{1}$. El SHF adquirido puede ser secundario a infecciones virales, enfermedades malignas, entidades autoinmunes, alteraciones metabólicas, inmunodeficiencias adquiridas o inmunosupresión asociada 
a trasplante de órganos sólidos o células madre. El pronóstico implica una alta mortalidad asociada a la enfermedad subyacente, las características del paciente, el momento del diagnóstico y la accesibilidad al tratamiento ${ }^{1-4}$.

El diagnóstico de este síndrome suele ser complejo debido a la variabilidad en la presentación, con criterios diagnósticos clínicos y de laboratorio. La complejidad en el diagnóstico radica en la presentación y en el solapamiento con las enfermedades de base. La presencia en la anatomía patológica de hemofagocitosis en la médula ósea es una herramienta diagnóstica, aunque no constituye un hallazgo patognomónico de esta entidad. De estar disponibles para medición, la ausencia de actividad NK y el aumento de IL-2 también son considerados parte del diagnóstico ${ }^{5,6}$.

Debido a las potenciales diferencias en los mecanismos fisiopatológicos de su producción, podrían existir diferencias en la forma de presentación clínica o de laboratorio del SHF asociado a distintas patologías. Identificar la clínica asociada a desarrollo del SHF podría ser de vital importancia para el reconocimiento del mismo de manera precoz $^{7-10}$. Debido al impacto de este síndrome y al potencialmente frecuente subdiagnóstico, el objetivo de este estudio fue describir las características, presentación clínica, causas y sobrevida asociadas al SHF.

\section{Materiales y Métodos}

Se realizó un estudio de cohorte dinámica retrospectiva de pacientes con sospecha o confirmación de SHF en el período 2003-2016, por revisión retrospectiva de historia clínica en el Hospital Italiano de Buenos Aires. El hospital es un centro de tercer nivel de alta complejidad, que cuenta con un repositorio de información único de cada paciente a través de una historia clínica electrónica (HCE). La HCE está orientada a problemas, con un servidor de terminología con tesauro local que asigna vocabulario controlado SNOMED CT a los problemas asignados a cada paciente. El estudio fue evaluado y aprobado por el comité institucional de ética de protocolos de investigación.

Como estrategia para la detección de los casos, se generó una lista con pacientes adultos (mayores a 17 años) que tuvieran cargado en la HCE el tér- mino SHF o sus sinónimos entre los problemas, diagnósticos principales o secundarios o cualquier campo de la epicrisis, que presentaran SHF o sus sinónimos en informes de anatomía patológica de biopsias de médula ósea u otros órganos y aquellos pacientes que reunieran una bicitopenia (hemoglobina menor a $9 \mathrm{~g} / \mathrm{dl}$, recuento de plaquetas menor a $100.000 / \mathrm{ml}$ y neutrófilos menor a $1.000 / \mathrm{ml}$ ) en combinación con ferritina elevada (ferritina mayor a $500 \mathrm{~g} / \mathrm{l}$ ) o hipertrigliceridemia (trigliceridos mayor a $265 \mathrm{mg} / \mathrm{dl}$ ) en el laboratorio. Las búsquedas se realizaron utilizando vocabulario controlado. Adicionalmente se añadieron a la búsqueda aquellos casos reportados por médicos del hospital con diagnóstico presuntivo de SHF.

Dos médicos entrenados revisaron la HCE de los pacientes detectados y definieron los casos sospechosos y los confirmados aplicando los criterios diagnósticos de la guía de la HLH-2004 $4^{10,11}$ que incluyen: fiebre (mayor o igual a $38,5^{\circ} \mathrm{C}$ ), esplenomegalia, citopenias con afectación de por lo menos dos de las tres series (hemoglobina menor a $9 \mathrm{~g} / \mathrm{dl}$, recuento de plaquetas menor a $100.000 /$ $\mathrm{ml}$ y neutrófilos menor a $1.000 / \mathrm{ml}$ ), ferritina mayor a $500 \mathrm{~g} / \mathrm{L}$, fibrinógeno menor a $150 \mathrm{mg} /$ dL y/o hipertrigliceridemia mayor a $265 \mathrm{mg} / \mathrm{dL}$, hemofagocitosis en médula u otros tejidos, baja actividad de células natural killer, niveles alto de receptor de IL-2. En nuestro hospital no contamos con la posibilidad de realizar las últimas 2 pruebas. Se incluyeron sólo los pacientes con sospecha o SHF confirmado según presentaran 4 o 5 criterios respectivamente. Se recuperó información sobre comorbilidades, características clínicas y de laboratorio de presentación, causas más frecuentes y evolución. Se clasificaron los SHF en 4 grupos de acuerdo a etiología: asociado a inmunosupresión, pacientes con cáncer, síndrome post infeccioso y de causa desconocida, de acuerdo a lo descrito en series previamente publicadas ${ }^{12,13}$. Con respecto al tratamiento de la enfermedad de base, se consideró como tratados a los pacientes con cáncer que recibieron quimioterapia, ganciclovir en pacientes infectados con citomegalovirus y tratamiento antimicrobiano específico en las infecciones bacterianas.

Cada paciente incluido fue seguido retrospectivamente desde la fecha de diagnóstico hasta la muerte, fecha de fin administrativo del estudio (16 de mayo de 2016), o pérdida al seguimiento. Se consideró el período de seguimiento desde la 
fecha de diagnóstico hasta la fecha de muerte, fecha de fin de seguimiento (última información disponible en la HCE) o fecha de fin de seguimiento administrativo.

Se presentan las variables categóricas como porcentajes y las variables continuas como media y desvío estándar (DE) o medianas e intervalos intercuartílicos (IIC) según distribución observada. Se estimó la sobrevida de los pacientes utilizando el método de Kaplan Meier. Se presenta la sobrevida a 7, 30, 60 días y a un año desde el diagnóstico, con sus intervalos de confianza de $95 \%$. Se evaluaron los factores asociados a mortalidad utilizando un modelo de regresión de riesgos proporcionales de Cox. El análisis estadístico se realizó utilizando el software Stata versión 14.0.

\section{Resultados}

Durante el período evaluado, se detectaron 15.219 potenciales casos, 8 con presencia de hemofagocitosis en muestra de anatomía patológica, 15.129 con ferritina mayor a 500; 64 por la combinación de bicitopenia e hipertrigliceridemia y 18 reportes de médicos. Posteriormente, se combinaron las variables ferritina mayor a $500 \mathrm{~g} / \mathrm{L}$, bicitopenia e hipertrigliceridemia, excluyéndose
15.163 pacientes por no reunir criterios mínimos. Se analizaron 56 potenciales casos de SHF, excluyéndose 29 pacientes que no cumplían con los criterios de sospecha o diagnóstico de SHF (Figura 1).

Se evaluaron las características basales de la población (Tabla 1). La mediana de edad fue de 51 años (rango de 18 a 87 años) y 16 (59,3\%) fueron hombres, siendo la comorbilidad más frecuente la insuficiencia renal. De los 27 casos analizados, 20 reunieron cinco criterios de SHF clasificándose como diagnóstico. Dentro de los parámetros de laboratorio, la anemia $(96,3 \%)$ y la plaquetopenia $(92,6 \%)$ seguidas de la ferritina elevada $(77,8 \%)$ fueron los hallazgos más frecuentes. La mediana de ferritina fue de 3.256 (IIC 25-75\% 1.600-5.000). El $88,9 \%$ de los pacientes presentó fiebre como parte del síndrome (Tabla 1).

La inmunosupresión fue la causa más frecuente de SHF con 14 pacientes (Tabla 2). Fueron categorizados como inmunosuprimidos pacientes trasplantados, aquellos que presentando cáncer hubieran realizado quimioterapia recientemente y consumidores crónicos de corticoides o inmunosupresores por cualquier causa. A su vez, dentro de este grupo 8 pacientes presentaron infección concomitante por citomegalovirus. Dentro del grupo de inmunosupresión se reportaron $7 \mathrm{ca}$ -

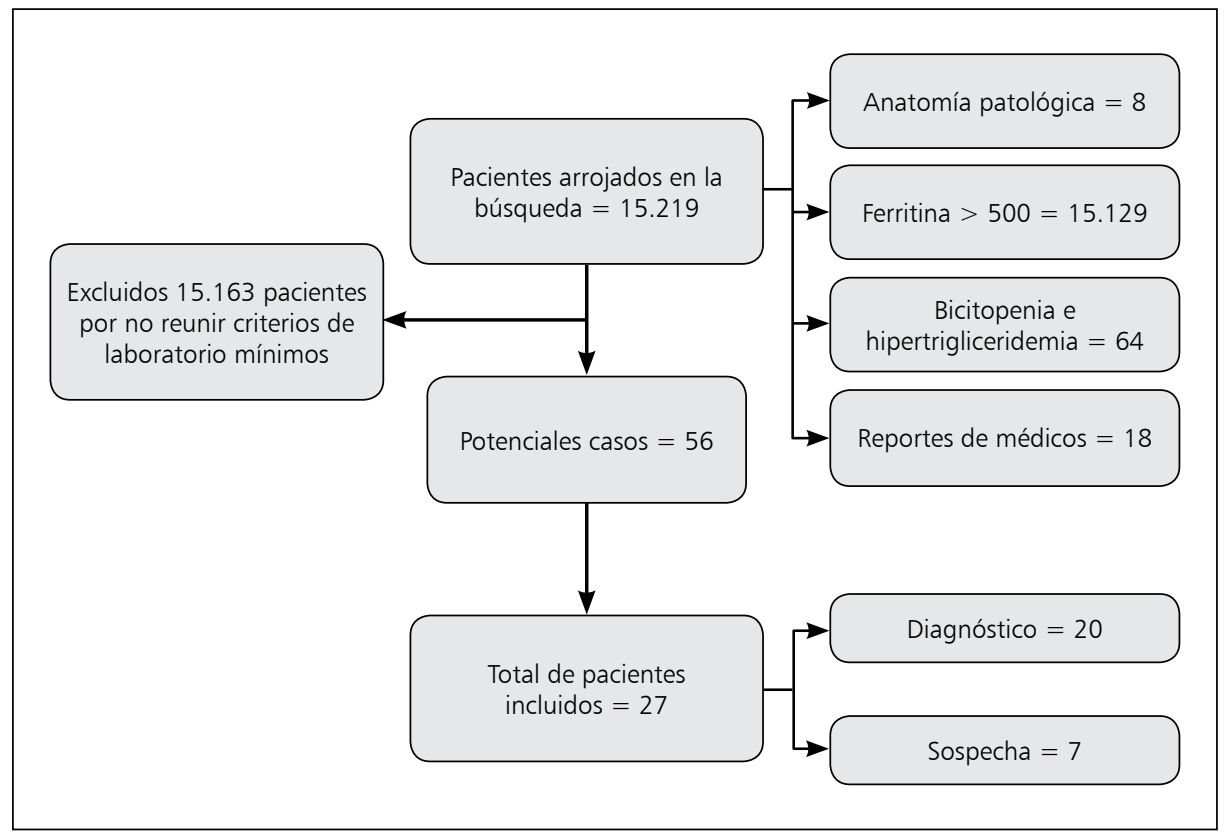

Figura 1. Diagrama de flujo. 
Tabla 1. Características de los pacientes con síndrome hemofagocítico. Pacientes $(n=27)$

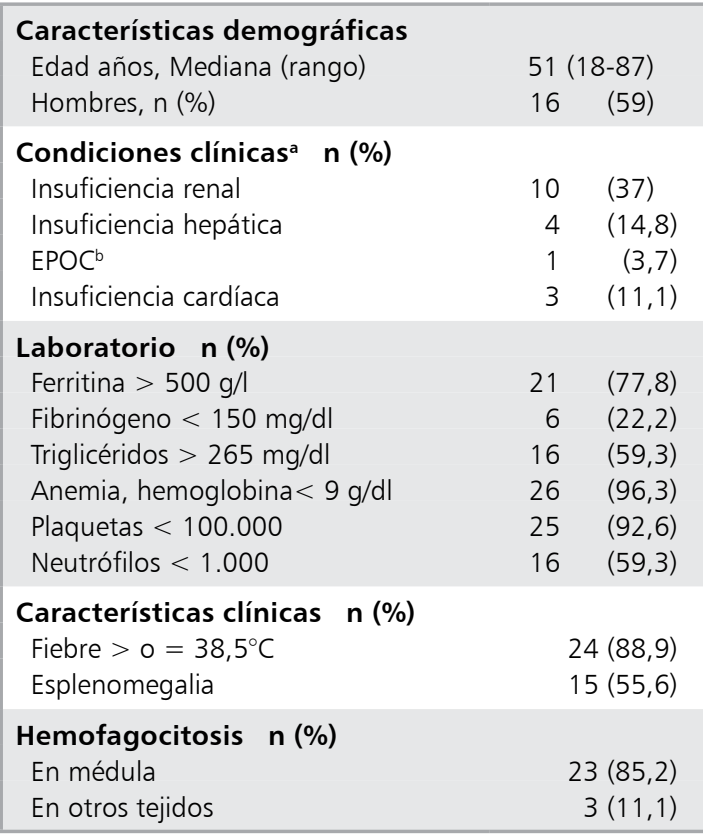

aDefinido como presente según registro positivo en historia clínica; 'benfermedad Pulmonar Obstructiva Crónica.

sos de trasplantados de médula ósea y 5 casos postquimioterapia. Los cánceres encontrados con mayor frecuencia fueron las enfermedades oncohematológicas, con 3 pacientes. Dentro del grupo de enfermedades infecciosas el aislamiento más comúnmente hallado fue el citomegalovirus en dos pacientes (Tabla 3 ).

Un total de 26 pacientes recibieron algún tipo de tratamiento para el SHF, los más frecuentes fueron el tratamiento de la enfermedad de base en 17 de ellos, seguido de gammaglobulina en 13 y corticoides en 9. La insuficiencia renal fue la complicación clínica más frecuente, con 9 pacientes, 6 pacientes sufrieron complicaciones hemorrágicas o trastornos de la coagulación y 5 insuficiencia cardíaca (Tabla 2).

De los 27 pacientes incluidos, 15 murieron y 7 se perdieron en el seguimiento, siendo 5 el número de pacientes que respondieron favorablemente al tratamiento. La mediana de tiempo de seguimiento fue de 29 días (0-365). La sobrevida estimada a los 7 días fue de 73,2\% (IC 95\% 51,8-86,2\%), a los 30 días 53,4\% (IC 95\% 32,7-70,3\%), a los 60
Tabla 2. Causas, tratamiento y complicaciones de los pacientes con síndrome hemofagocítico. Pacientes $(\mathbf{n}=\mathbf{2 7})$

\begin{tabular}{|c|c|c|}
\hline \multicolumn{3}{|l|}{ Causas $\mathrm{n}(\%)$} \\
\hline Inmunosupresión & 14 & $(51,9)$ \\
\hline Cáncer & 5 & $(18,5)$ \\
\hline Post-infeccioso & 6 & $(22,2)$ \\
\hline Desconocido & 2 & $(7,1)$ \\
\hline Tratamiento $\mathbf{n}(\%)$ & & \\
\hline Gammaglobulina & 13 & $(48,2)$ \\
\hline Corticoides & 9 & $(33,3)$ \\
\hline Quimioterapia (Etopósido) & 3 & $(11,1)$ \\
\hline Enfermedad de base & 17 & (63) \\
\hline Plasmaféresis & 1 & $(3,7)$ \\
\hline Complicaciones $^{\mathrm{b}}$ n (\%) & & \\
\hline Insuficiencia renal & 9 & $(33,3)$ \\
\hline Insuficiencia hepática & 2 & $(7,4)$ \\
\hline Insuficiencia cardíaca & 5 & $(18,5)$ \\
\hline Shock cardiogénico & 1 & $(3,7)$ \\
\hline Shock séptico & 2 & $(7,4)$ \\
\hline Anemia & 3 & $(11,1)$ \\
\hline Hemorragia & 4 & $(14,8)$ \\
\hline Trastornos de la coagulación & 2 & $(7,4)$ \\
\hline Enfermedad tromboembólica & 1 & $(3,7)$ \\
\hline
\end{tabular}

${ }^{a}$ Quimioterapia en caso de pacientes con diagnóstico de cáncer, Ganciclovir en pacientes infectados con Citomegalovirus y tratamiento antimicrobiano específico en las infecciones bacterianas. 'befinido como presente según registro positivo en historia clínica.

Tabla 3. Clasificación por causas de los pacientes con síndrome hemofagocítico. Pacientes $(n=27)$

\begin{tabular}{ll|}
\hline Inmunosupresión & \\
Trasplante de médula ósea & 7 \\
Pacientes con cáncer post-quimioterapia & 5 \\
Transplantado cardíaco & 1 \\
Transplantado bipulmonar & 1 \\
Asociado a infecciones & \\
Citomegalovirus & 2 \\
Criptococosis & 1 \\
Virus de la Inmunodeficiencia Humana & 1 \\
Epstein Barr Virus & 1 \\
Leptospirosis & 1 \\
Asociado a cáncer & \\
Linfoma no Hodgkin & 1 \\
Leucemia mieloide aguda M5 & 1 \\
Leucemia linfoblástica & 1 \\
Sarcoma de Kaposi & 1 \\
Cáncer de riñón & 1 \\
Causa desconocida & 2 \\
\hline
\end{tabular}




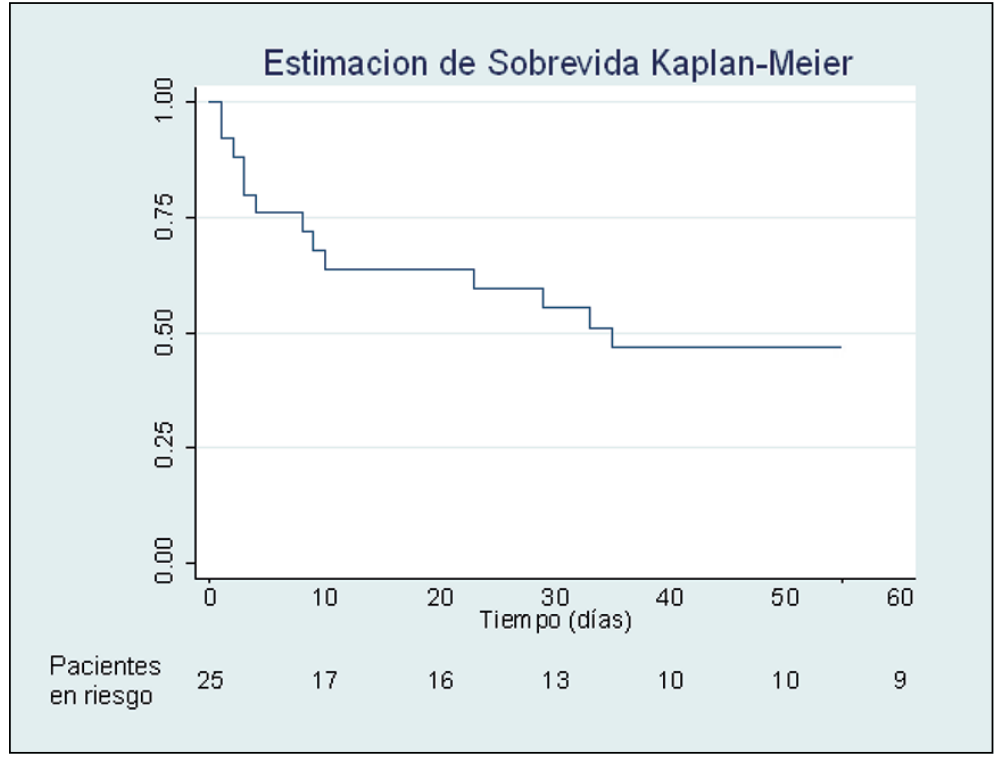

Figura 2. Gráfico de sobrevida de Kaplan-Meier a 60 días. días 45,2 (IC 95\% 25,5-62,9\%) y al año 38,7\% (IC 95\% 19,2-57,9\%). La mediana de tiempo al evento fue de 33 días (Figura 2).

En el análisis de supervivencia (Tabla 4), se encontró una asociación significativa en los pacientes con insuficiencia renal con un HR de 3,4 (IC95\% 1,2-9,9 p 0,025). En relación a los tratamientos instaurados, el tratamiento de la enfermedad de base fue el único que demostró ser protector HR de 0,32 (IC95\% 0,1-0,9 p 0,046).

\section{Discusión}

Nuestro estudio describe las características principales, causas más frecuentes y mortalidad en pacientes con SHF. Dentro de las características demográficas observamos una media de edad menor a la reportada por estudios previos ${ }^{12,13}$, pero igual a la descrita recientemente por Zhang $\mathrm{y} \mathrm{col}^{14}$. La inmunosupresión resultó ser la causa más frecuente en nuestra cohorte, seguida del SHF post-infeccioso, lo que difiere de estudios previos, donde las causas más frecuentemente descritas fueron el cáncer y la infecciosa ${ }^{14}$. Esta diferencia puede residir, en parte, en la forma de clasificación de los pacientes, dado que se ha considerado dentro del grupo inmunosupresión a
Tabla 4. Hazard Ratio para muerte

\begin{tabular}{|lcc|}
\hline & $\begin{array}{c}\text { Hazard Ratio } \\
\text { (IC95\%) }\end{array}$ & $\mathbf{p}^{\mathbf{a}}$ \\
\hline $\begin{array}{l}\text { Factores demográficos } \\
\text { Edad }\end{array}$ & $1(0,97-1,03)$ & 0,862 \\
Sexo & $0,7(0,3-2,2)$ & 0,583 \\
Causas & & \\
Cáncer & 1 (categoría de referencia) \\
Post-infeccioso & $18,1(1,9-177,7)$ & 0,013 \\
Inmunosuprimido & $3,5(0,4-28,6)$ & 0,234 \\
Desconocido & $7,3(0,4-127,2)$ & 0,173 \\
Tratamiento & $1,1(0,4-3,2)$ & 0,871 \\
Gammaglobulina & $0,9(0,3-3)$ & 0,895 \\
Corticoides & $1,3(0,3-5,7)$ & 0,751 \\
Quimioterapia & $0,3(0,1-0,98)$ & 0,046 \\
Enfermedad de base & & \\
Comorbilidades & $3,4(1,2-9,9)$ & 0,025 \\
Insuficiencia renal & $2,4(0,5-11,2)$ & 0,25 \\
\hline Insuficiencia cardíaca & & \\
\hline
\end{tabular}

p modelo de regresión logística.

pacientes trasplantados de órgano sólido o médula ósea y pacientes con cáncer postquimioterapia. A su vez, esto podría deberse a un mayor número de pacientes sometidos a trasplante, este último subgrupo se encuentra menos caracterizado en la 
literatura. Adicionalmente, el número de casos sin causa aparente fue mayor en reportes previos ${ }^{13}$.

El tratamiento para el SHF consiste en el tratamiento de la enfermedad de base asociado a la terapia adyuvante con corticoides, reservándose el uso de quimioterapia con etopósido para aquellos casos con inestabilidad clínica o progresivos ${ }^{11,15}$. Luego del tratamiento de la enfermedad de base, el uso de gammaglobulina constituyó la terapéutica más frecuente. A diferencia de estudios previos, donde más de la mitad de los pacientes fueron tratados exclusivamente con corticoides, en nuestra cohorte sólo 2 pacientes recibieron este tratamiento como monoterapia ${ }^{1,13,16}$.

Llamativamente, la mortalidad del SHF sólo se vio asociada positivamente al tratamiento de la enfermedad de base, y no a la causa como fue descrito en estudios previos, aunque no podemos descartar que pudieron haber recibido tratamiento los pacientes que vivieron más tiempo ${ }^{13,16}$. El único determinante negativo fue la falla renal al momento del diagnóstico. Creemos que el bajo número de pacientes con SHF secundario a inmunosupresión aún no nos permite observar diferencias con las otras etiologías. Se requiere estudios con mayor cantidad de pacientes para determinar si este subgrupo tiene una evolución $y$ un tratamiento independiente del resto y cuanta mortalidad agrega el síndrome hemofagocítico a la enfermedad de base.

El pronóstico de los pacientes con SHF es ominoso. Ya desde del protocolo original HLH-1994, la sobrevida a 5 años era de $54 \%$ y resultados similares fueron publicados en estudios posteriores ${ }^{12,14}$. Pocas series reportan sobrevida estimada. Si bien en nuestra cohorte la sobrevida fue menor que en otras series reportadas, nuestros intervalos de confianza se superponen con las curvas de sobrevida estimada. El síndrome hemofagocítico presenta mayor mortalidad aguda y la escasa cantidad de casos en seguimiento hacen que las estimaciones de sobrevida sean muy imprecisas, con intervalos de confianza muy amplios. No podemos descartar que la menor sobrevida observada en nuestra cohorte pudiera deberse a un diagnóstico más tardío en nuestro medio o a mayor gravedad de los pacientes en seguimiento. Por otro lado, existe la posibilidad de que este resultado manifieste el mal pronóstico que se cree tienen los SHF secundarios a inmunosupresión, que predominaron en nuestro estudio ${ }^{8}$.
La baja frecuencia de este síndrome dificulta la realización de estudios prospectivos, por lo que pensamos que este acercamiento de tipo retrospectivo es la estrategia más adecuada. Debido a la presentación sindromática de esta patología, se decidió utilizar estrategias de búsqueda múltiples para reducir al mínimo la probabilidad de pérdida de casos. Pese a esto, la detección de pacientes con SHF pudo haber sido incompleta. Sin embargo, la HCE es un repositorio único de información que permite obtener datos de alta calidad que nos permite obtener información confiable de patologías poco frecuentes.

Adicionalmente, durante la búsqueda de pacientes con SHF hallamos un número importante de pacientes con hemofagocitosis en médula ósea sin síndrome clínico asociado. Este subgrupo de paciente (no bien caracterizados en la bibliografía) podrían tener características particulares, y asociaciones con patologías no descritas actualmente. Creemos que este hallazgo debe ser jerarquizado, y estudios posteriores podrían ayudarnos a comprender mejor este fenómeno.

Los criterios moleculares utilizados habitualmente en el diagnóstico de SHF no han podido ser descritos, debido a la ausencia de dichas técnicas en nuestro ámbito ${ }^{10}$. Esto podría generar una potencial pérdida de casos de pacientes con SHF, sin embargo, al no tratarse de técnicas disponibles universalmente, creemos que nuestros resultados podrían extrapolarse a la mayoría de centros privados y públicos en nuestro país que tampoco disponen de técnicas moleculares ${ }^{17-19}$.

En conclusión, hemos podido describir las características clínicas y de laboratorio, las causas, el tratamiento y la mortalidad de un síndrome de baja frecuencia y difícil diagnóstico. Creemos importante la identificación de las potenciales causas de SHF ya que el pronóstico se podría relacionar con el tratamiento de la enfermedad de base. Consideramos que el estudio de la fisiopatología de este síndrome permitirá una mejor comprensión y tratamiento del mismo.

\section{Referencias}

1. Celkan T, Berrak S, Kazanci E, Ozyürek E, Unal S, Uçar $\mathrm{C}$, et al. Malignancy-associated hemophagocyticlymphohistiocytosis in pediatric cases: a multicenter study from Turkey. Turk J Pediatr 2009; 51: 207-13.

2. Zhang L, Zhou J, Sokol L. Hereditary and acquired 
hemophagocyticlymphohistiocytosis. Cancer Control 2014; 21: 301-12.

3. Janka GE. Familial and acquired hemophagocyticlymphohistiocytosis. Eur J Pediatr 2007; 166: 95-109.

4. Bode SF, Lehmberg K, Maul-Pavicic A, Vraetz T, Janka $\mathrm{G}$, Stadt UZ, et al. Recent advances in the diagnosis and treatment of hemophagocyticlymphohistiocytosis. Arthritis Res Ther 2012; 14: 213.

5. Janka GE, Lehmberg K. Hemophagocytic syndromes-an update. Blood Rev 2014; 28: 135-42.

6. Trottestam H, Horne A, Aricò M, Egeler RM, Filipovich $\mathrm{AH}, \mathrm{Gadner} \mathrm{H}$, et al. Chemoimmunotherapy for hemophagocytic lymphohistiocytosis: long-term results of the HLH-94 treatment protocol. Blood 2011; 118: 4577-84.

7. Machaczka M, Sydor W, Rucinska M, Szostek M, Musia J. Autoimmune-Associated Hemophagocytic Syndrome/ Macrophage Activation Syndrome. Autoimmune Disorders - Current Concepts and Advances from Bedside to Mechanistic Insights. 2011.

8. Karras A, Thervet E, Legendre C. Hemophagocytic syndrome in renal transplant recipients: report of 17 cases and review of literature. Transplantation 2004; 77 : 238-43.

9. Otrock ZK, Eby CS. Clinical characteristics, prognostic factors, and outcomes of adult patients with hemophagocytic lymphohistiocytosis. Am J Hematol 2015; 90: 220-4.

10. Henter J-I, Jan-Inge H, AnnaCarin H, Maurizio A, Maarten Egeler R, Filipovich AH, et al. HLH-2004: Diagnostic and therapeutic guidelines for hemophagocytic lymphohistiocytosis. Pediatr Blood Cancer 2007; 48: 124-31.

11. Jordan MB, Allen CE, Weitzman S, Filipovich AH, McClain KL. How I treat hemophagocytic lymphohis- tiocytosis. Blood 2011; 118: 4041-52.

12. EgüesDubuc C, Dubuc CE, Cáceres VA, Ecenarro MU, Aguirre NE, Rubio IH, et al. Secondary Macrophage Activation Syndrome Due to Autoimmune, Hematologic, Infectious and Oncologic Diseases. Thirteen Case Series and Review of the Literature. Reumatología Clínica (English Edition) 2015; 11: 139-43.

13. Oto M, Yoshitsugu K, Uneda S, Nagamine M, Yoshida M. Prognostic Factors and Outcomes of Adult-Onset Hemophagocytic lymphohistiocytosis: A Retrospective Analysis of 34 Cases. Hematol Rep 2015; 7: 5841.

14. Zhang Z, Wang J, Ji B, Greenwood T, Zhang Y, Wang $\mathrm{Y}$, et al. Clinical presentation of hemophagocytic lymphohistiocytosis in adults is less typical than in children. Clinics 2016; 71: 205-9.

15. Schram AM, Berliner N. How I treat hemophagocytic lymphohistiocytosis in the adult patient. Blood 2015; 125: 2908-14.

16. Reddy S, Rangappa P, Kasaragod A, Kumar AS, Rao K. Haemophagocytic lymphohistiocytosis (HLH): case series in tertiary referral hospital over three years. J Assoc Physicians India 2013; 61: 850-2.

17. Young P, Peroni J, Finn BC, Venditti JE, Preiti V, Bullorsky E, et al. Síndromehematofagocítico: Reporte de cuatro casos y revisión de la literatura. Rev Med Chile 2011; 139: 224-9.

18. Kleinert MM, Garate G, Osatnik J, Cicco J, Hunter B, Soria EJ. [Reactive hemophagocytic syndrome in critical care patients. Report of 4 cases]. Medicina 2007; 67: 4952.

19. Mahuad CV, Garate GM, Vicente Reparaz M de L, Casali C, Del Olmo M, Bolgiani A. [Secondary hemophagocytic syndrome due to recurrent infections in a severely burned patient]. Medicina 2013; 73: 255-8. 